\title{
Experimental studies of two-phase liquid metal-gas chain flow with ultrasonic echo pulse method and in the magnetic field of permanent magnets
}

\author{
Artur Andruszkiewicz, ${ }^{1, *}$, and Kerstin Eckert ${ }^{2}$ \\ ${ }^{1}$ Wrocław University of Science and Technology, Wybrzeże Stanisława Wyspiańskiego 27, 50-370 Wrocław, Poland \\ ${ }^{2}$ Technische Universitätv Dresden, Faculty of Mechanical Science and Engineering, George-Bähr- Strasse 3c, 01069 Germany
}

\begin{abstract}
Metallurgical processes of refining and degassing liquid metals are related to the blowing-in neutral gasses like for example argon. Effectiveness of the process depends on the flowing gas parameters like: dimensions, velocity, distribution amd gas phase flow zone. These parameters can be changed under the influence of outside magnetic field and formed by it a Lorentz' force. In order to get full information of rising bubbles essential are certain measuring methods. Among many of these the ultrasonic echo pulse method is likely to be the most intensive developed in the past few years. That method enables estimation of gas phase flow parameters in a range from individual bubble flow up to full continuous gas flow at the inlet nozzle. In this paper a measurement principle has been described for an ultrasonic echo method and there also submitted and discussed results of obtained results for diphase flow of GaInSn-argon.
\end{abstract}

\section{Introduction}

The two-phase liquid metal-gas flow appears in many technological processes like in metallurgical process of after-furnace steel refining. The process of liquid metal degassing, purification or enrichment are directly related to argon blow-in. A refining efficiency depends on the dimension of rising bubbles, its contact time with metal, the rise zone, and the flow stream of bubbles. A proper recognition of these parameters enables optimization as well as control of metallurgical processes. In order to get a complete knowledge of the rising gas bubbles an experimental studies of two-phase liquid metal-gas flow are essential. Among many methods of investigation two-phase flows recently more and more important becomes the ultrasonic method. For example, the ultrasonic echo pulse method is presently used by TU Dresden for identification of two-phase liquid metal-gas flow parameters in their project studies on "Elektromagnetische Stromungsbeeinflussung in Matallurie, Kristallzuchtung und Elektrochemie" no SFB 609.

The aim of the article is to present selected research results: rise velocity of bubbles, its creation frequency, dimensions and flow zone in the liquid metal using the echo pulse methopd. The method has been elaborated for a container filled with liquid metal $\mathrm{Ga}^{68} \mathrm{In}^{20} \mathrm{Sn}^{12}$ where the argon gas phase was blow-in through a nozzle. Measurements were also done in magnetic field of permanent magnets having magnetic induction of $B=300$ $\mathrm{mT}$. Magnetic field influences the rise velocity and the final velocity of gas bubbles, the movement trajectory and the zone of gas phase flow as well as the drag coefficient value. The article also compare the gas phase flow parameters in the liquid metal in motion, in the magnetic field and without the magnetic field.

\section{Ultrasonic echo pulse method - the measurement principle}

Among many methods of two-phase liquid metal-gas flow studies the biggest significance have got the non invasive techniques. Its basic advantages are no interference with the flow, no perturbations of velocity profile distribution and no changes of position distribution and diameter of rising bubbles. A non invasive techniques enable assign the basic two-phase flow parameters practically in a real time. In the studies are used ultrasonic Doppler's methods (UDV) [1-3], tomography techniques: positron-emission PET and roentgen CT [4] as well as methods utilizing eddy current and magnetic sensors [5-6]. The ultrasonic echo pulse method which can be used in identification of twophase liquid metal-gas flows it is not yet fully developed and propagated comparing to the other above mentioned methods. That method is most widely used in non invasive material investigation - ultrasonic defectoscopy. It is a primary way of material fault detection also giving the localization, dimensions and space orientation of the defects. Presuming a gas phase as flow discontinuity than the echo pulse method can be utilized to estimate characteristic parameters of two-phase liquid metal-gas flow.

The primary work pertaining to estimation of liquid metal-gas flow parameters with utilized echo method

\footnotetext{
* Corresponding author: artur.andruszkiewicz@pwr.edu.pl
} 
and ultrasonic defectoscope is contributed to Hoffman \& Kaiser of TU Dresden. They used two or one ultrasonic heads made by Krautkrammer and sampled signals with digital oscilloscope. The measurement tests done with one ultrasonic head were not difficult (for estimation of volumetric share) but in a case when two heads were used for velocity measurements it was necessary to set an appropriate correlation between ultrasonic pulse frequency and signal recording time [7], and also properly set the signal recording time in order to record all of the flowing gas bubbles. The study results were presented in papers [5-6].

The measuring principle used in echo pulse method, shown on Fig.1, is described in detail in [8]. Short pulses transmitted from ultrasonic head goes through a connection layer (mostly of Vaseline) and front wall of a container than are reflected from gas bubbles moving in liquid and being in the ultrasonic area.

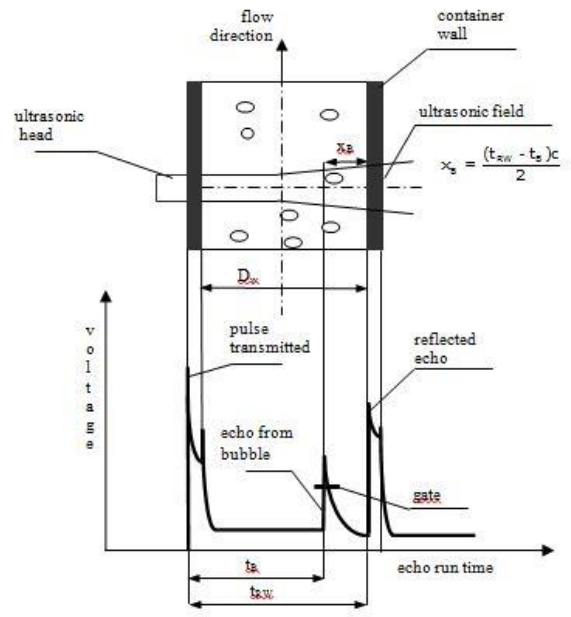

Fig. 1. The measuring principle used in echo pulse method: $t_{B}-$ run time for an echo reflected from gas bubbles, $\mathrm{t}_{\mathrm{RW}}-\mathrm{echo}$ run time for an echo reflected from back wall of container, $c-$ ultrasonic wave velocity in liquid, $x_{\mathrm{B}}-\mathrm{a}$ distance between a bubble and inner part of container back wall.

Ultrasonic pulses reflected from bubbles return as echo back to the ultrasonic head which operate in a transmitter-receiver mode. Part of the transmitted pulses reach the back wall of container and reflected goes back to ultrasonic head. Calculating the time difference between pulses reflected from gas bubbles and container back wall the process characteristic may be presented as a voltage-time curves on ultrasonic defectoscope. It is an A type presentation shown on a Fig.1. The ultrasonic defectoscope records only signals overcrossing a set level, mostly $20 \%$ of the maximum screen scale level. For this value the run time for a wave patch from transmitter to reflector and back is calculated. Knowing the wave velocity in the container wall and tested medium a distance from the reflector may be calculated. The upper part of Fig.1. Shows the distance $x_{\mathrm{B}}$ of the bladder from the back wall of the tank. Its determination requires only the knowledge of the velocity of the ultrasonic wave in the medium. The repetition frequency of ultrasonic pulses should be set in such a way that the echo reflected from back wall of the container should be recorded before sending a next pulse.

\section{The testing bench - a measurement realization}

The test studies were executed on a test bench as shown on Fig. 2.

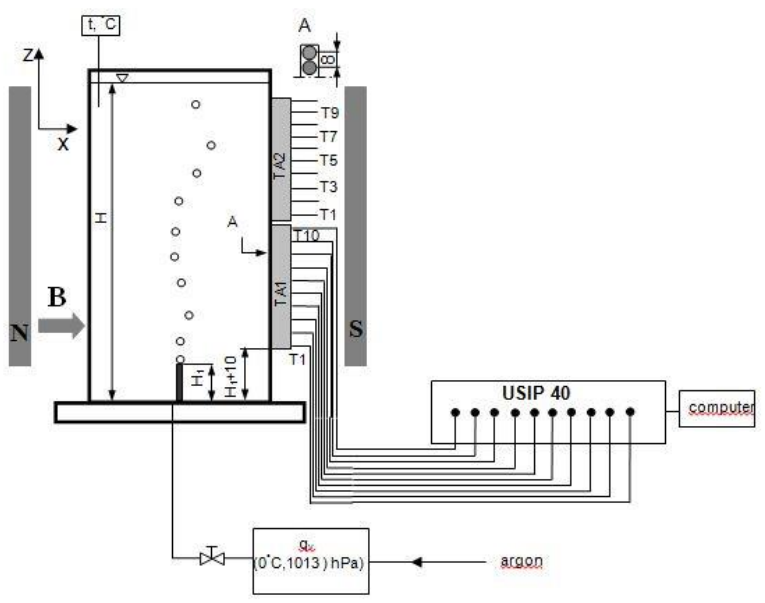

Fig. 2. A testing bench diagram.

A container with a diameter of $D_{\mathrm{w}}=80 \mathrm{~mm}$ was filled up with liquid metal GaInSn to the height of $H=$ $210 \mathrm{~mm}$ (with density of $\rho_{\mathrm{FM}}=6361 \mathrm{~kg} / \mathrm{m}^{3}$, surface tension $\sigma_{\mathrm{FM}}=0.533 \mathrm{~N} / \mathrm{m}$ and temperature $t=20^{\circ} \mathrm{C}$ ). Argon in the form of gas bubbles has been blow-in through a nozzle with a height of $20 \mathrm{~mm}$ and aperture diameters of $0.5 \mathrm{~mm}, 0.9 \mathrm{~mm}$ and $1.2 \mathrm{~mm}$, depending on the test number. The volumetric argon gas flow $q_{\mathrm{v}}$ has been measured with thermo-sensor flow meter (Mas-Flo, MKS Instruments) class 1, with ranges: for single rising bubbles $10 \mathrm{sccm}$, and for a chain bubbles $100 \mathrm{sccm}$. The ultrasonic head (TA1) was fixed $10 \mathrm{~mm}$ above the argon inlet nozzle. The head consisted 10 sensors having diameter of $5 \mathrm{mms}$ each with frequency $15 \mathrm{MHz}$, spaced at $8 \mathrm{~mm}$ (the total distance between sensors has been 72 $\mathrm{mm}$ ), and operated in transmission-receiving mode. The created ultrasonic zone in liquid metal GaInSn has a characteristic length of local field $l_{\mathrm{o}}=34.3 \mathrm{~mm}$ and opening angle of beam $\varphi \approx 2.6^{\circ}$.

Signals from rising gas bubbles were recorded by means of two measuring heads installed one above the other (TA1, TA2), that enabled to measure bubbles velocity and its changes on the rise path ca. $160 \mathrm{~mm}$. Ultrasonic heads were alternatively connected to a 10 channel defectoscope USIP 40 Box controlled by computer with a software UltraPROOf (by Krautkramer). In the tests only the measurement of echo run time from the rising bubbles were utilized because its very low signal-to-noise ratio that allows the exact estimation of gas bubble contours. The echo overshoot time resolution has been $2.5 \mathrm{~ns}$, that equals to an alteration of bubble position in a liquid metal of ca. $0.004 \mathrm{~mm}$. A pulse repetition frequency was set to a level of $14286 \mathrm{~Hz}$, the highest setting value for 10 ultrasonic sensors, and signal amplification was set in 
such a way that the amplitude of signals over passed $20 \%$ of the level shown on Fig.1. The signal sampling period equals $0.7 \mathrm{~ms}$. The recording period was set at $360 \mathrm{~s}$, according to a principle proved by Sommerlatt [7] that to get representative results a sampling period have to be longer than 2 minutes. For such recording time it is possible to record even several thousand rising bubbles and exactly estimate its rise velocity and rise zone, depending on the creation frequency of argon bubbles.

Likewise measurements of rising bubbles in a permanent magnetic field has been done with magnets of average induction $B=300 \mathrm{mT}$ (by Teslameter model 5180). Two magnets $160 \times 100 \times 50$ were spaced at 115 $\mathrm{mm}$.

The resultant Lorentz' force in a magnetic field of induction $\mathrm{B}$ equals to:

$$
\mathbf{F}=\mathbf{j} \times \mathbf{B}=\sigma_{\mathrm{el}}(\mathbf{w} \times \mathbf{B}) \times \mathbf{B}
$$

where: $\mathbf{j}$ - current density, $\mathbf{w}$ - liquid metal velocity, $\sigma_{\mathrm{el}}-$ electric conductivity.

Drawing no 3 shows the measuring bench container with ultrasonic heads in magnetic field as well as directions and sense of Lorentz' force.
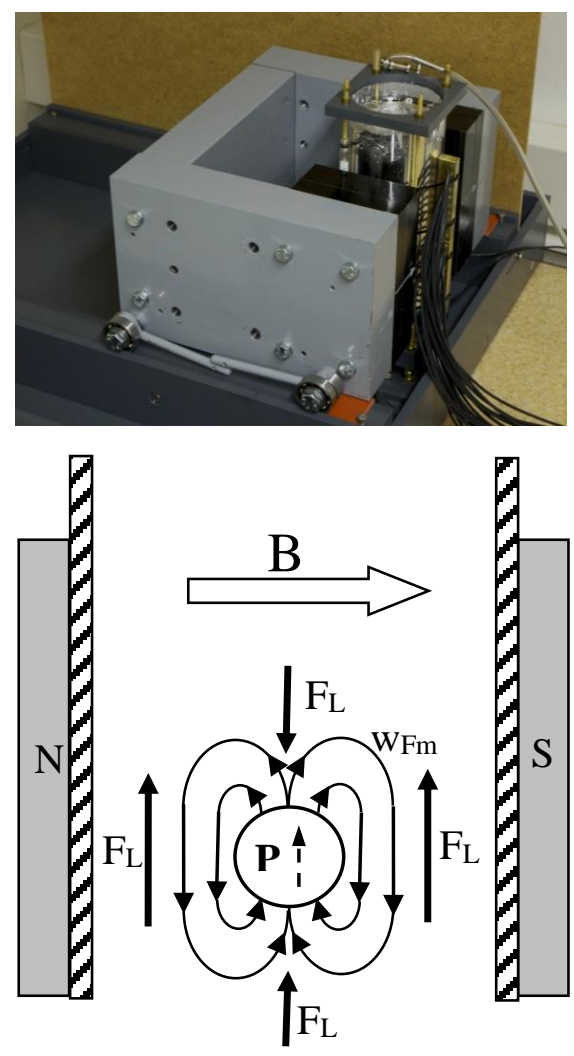

Fig. 3. Testing (measuring) bench in a magnetic field and Lorentz' forces acting on liquid metal in magnetic field: $w \mathrm{Fm}-$ liquid metal velocity, $\mathrm{P}-$ gas bubble rising up vertically.

\section{The results of studies and discussion}

\subsection{Rise velocity of bubbles}

A typical measuring signal of rising bubbles of echo run time method is shown on Fig.4.

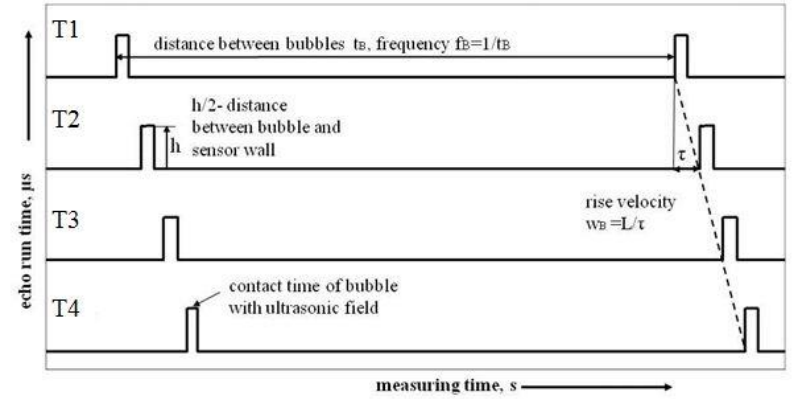

Fig. 4. Measuring signal of echo run time method.

The velocity $w_{\mathrm{B}}$ of the bubble (vertical component of velocity) was calculated by measuring the bubble time $\tau$ on the $L$ road between two successive ultrasonic sensors. Fig.5. shows exemplary results of bubble velocity measurements from the height above the argon inlet nozzle for $B=0 \mathrm{mT}$ and $B=300 \mathrm{mT}$. Fig.6. shows the dependence of average speed on the flow of argon volume calculated for the condition at the inlet nozzle also for $B=0 \mathrm{mT}$ and $B=300 \mathrm{mT}$.

Starting from the volume flow of argon at the nozzle $q_{\mathrm{v}}=1.8 \mathrm{l} / \mathrm{h}$, the velocity of the bubbles in the magnetic field is lower than the velocity for $B=0 \mathrm{mT}$. Differences in velocities are increasing with increasing gas flow. The flow value $q_{\mathrm{v}}=1.8 \mathrm{l} / \mathrm{h}$ can be taken as the beginning of the influence of Lorentz force on the velocity of rising bubbles.

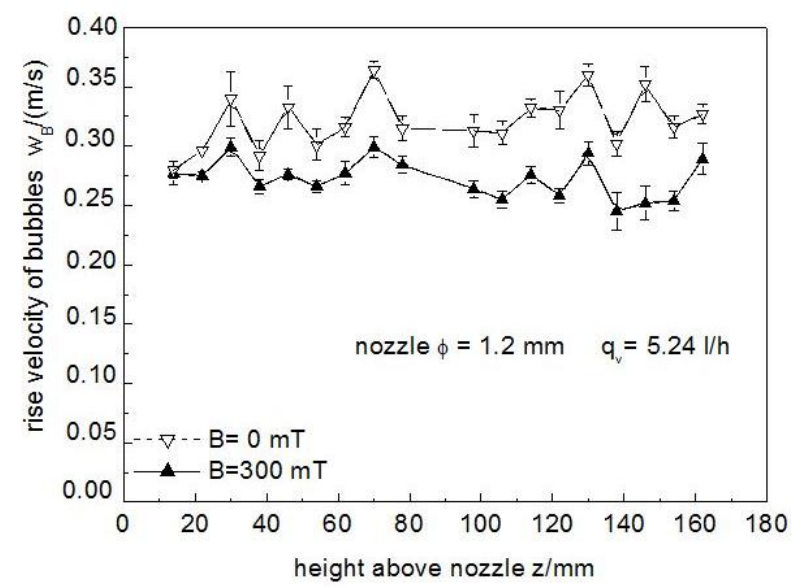




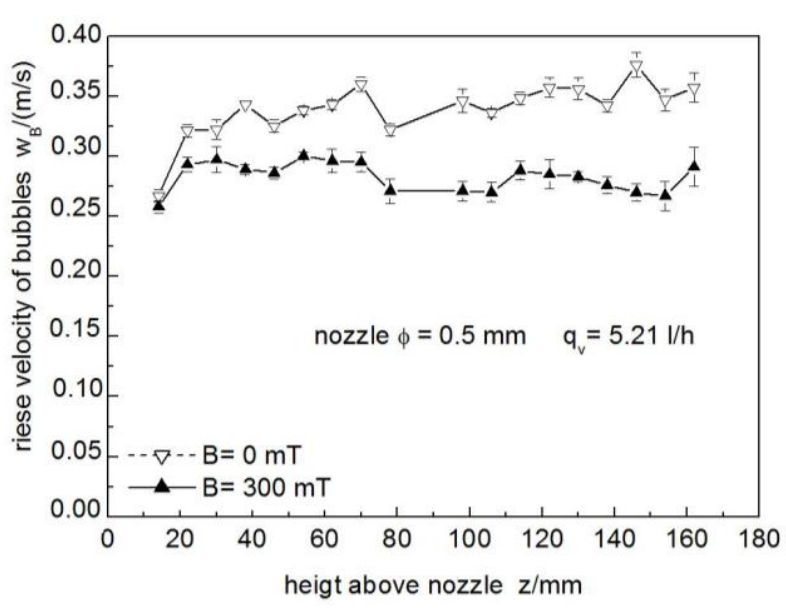

Fig. 5. Rise velocity of bubbles for $B=0 \mathrm{mT} \& B=300 \mathrm{mT}$.

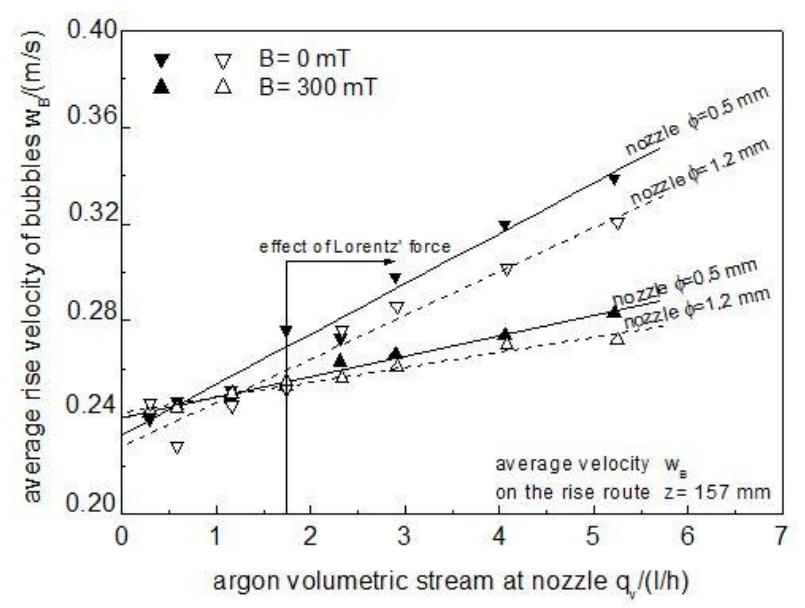

Fig. 6. Rise velocity of bubbles as a function of argon volumetric stream at nozzle.

\subsection{The creation frequency of bubbles and its dimension}

The process of gas bubble creation is difficult to describe theoretically and because of it to estimate the frequency and dimension of bubbles empirical approximate equations are used. The basic equation to calculate the frequency of bubble creation is given in a work [9]:

$$
f_{\mathrm{B}}=3.82 \cdot 10^{-5} \cdot\left(\mathrm{g}^{0.75} \cdot q_{\mathrm{v}} /\left(d_{\mathrm{o}}^{0.75} \cdot \mathrm{v}^{1.5}\right)\right)
$$

where: $\mathrm{v}$ - kinematic viscosity coefficient, $q_{\mathrm{v}}-$ gas volumetric stream at nozzle.

The authors of study [10] pointed out that utilization of the above mentioned equation for a nozzle of $0.5 \mathrm{~mm}$ diameter and water-air flow gives errors of more than 100\%. On a Fig. 7 are presented experimental study results showing a relationship of bubble creation frequency as a function of argon stream volume at nozzle. The results were calculated with Fourier quick transform FFT of measured signal. As the studies shown, in practice a magnetic field does not exert an influence on bubble creation frequency changes.

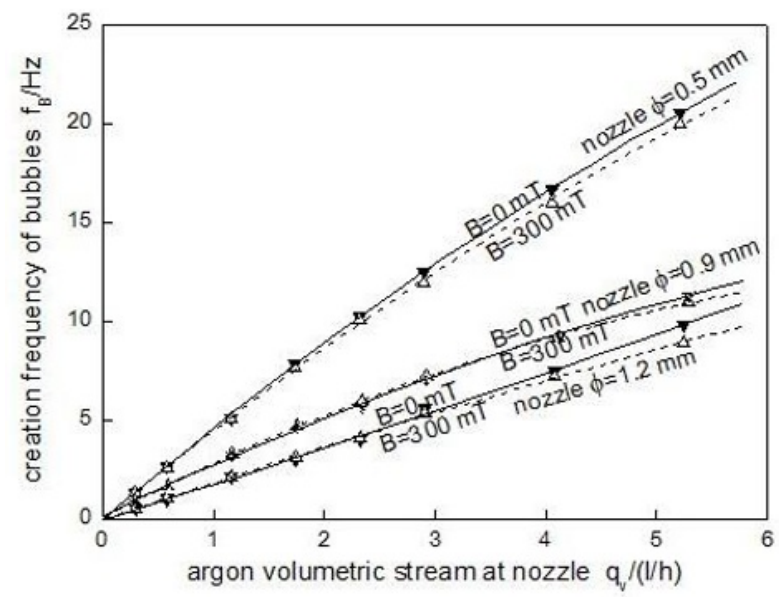

Fig. 7. Creation frequency of argon bubbles as a function of gas volumetric stream at nozzle for $B=0 \mathrm{mT} \& B=300 \mathrm{mT}$.

The frequency difference between $B=0 \mathrm{mT}$ and $B=$ $300 \mathrm{mT}$ exists for argon volumetric flow greater than 6 $1 / \mathrm{h}$ and nozzles of $0.5 \& 1.2 \mathrm{~mm}$. Anyhow they does not exceed $10 \%$ and seems to be of measurement errors origin. The diameter of rising bubble $d_{B}$ is calculated with Mersmann's equate in a form of:

$d_{\mathrm{B}}=\left\{3 \cdot \sigma \cdot d_{\mathrm{o}} /(\mathrm{g} \cdot \rho)+\left(\left(3 \cdot \sigma \cdot d_{\mathrm{o}} /(\mathrm{g} \cdot \rho)\right)^{2}+\left(15 \cdot q_{\mathrm{v}}{ }^{2} \cdot d_{\mathrm{o}} / \mathrm{g}\right)\right)^{1 / 2}\right\}^{1 / 3}$

This equate has been delivered on the base of force balance influencing an individual spherical rising gas bubble in motionless liquid. A gas bubbles are introduced into container through a nozzle with a diameter of $\mathrm{d}_{\mathrm{o}}$, and gas stream rises it up $q_{\mathrm{v}}$.

The above given equation were used by Kaiser [5-6] to estimate diameter of rising gas bubbles in mercury, with gas flow stream $q_{\mathrm{v}}=3.6 \mathrm{l} / \mathrm{h}$. From among many others correlation $\mathrm{s}$ it is worth to mention Kantarcia [11] equations for calculating diameter of bubbles moving in barbotage columns.On a Fig.8 is presented relationship of bubble average equivalent diameter $\mathrm{d}_{\mathrm{B}}$ on gas volumetric stream for a nozzle $q_{\mathrm{v}}$. It is calculated by means of:

$$
d_{\mathrm{B}}=\left(6 \cdot q_{\mathrm{v}} /\left(\pi \cdot f_{\mathrm{B}}\right)\right)^{1 / 3}
$$

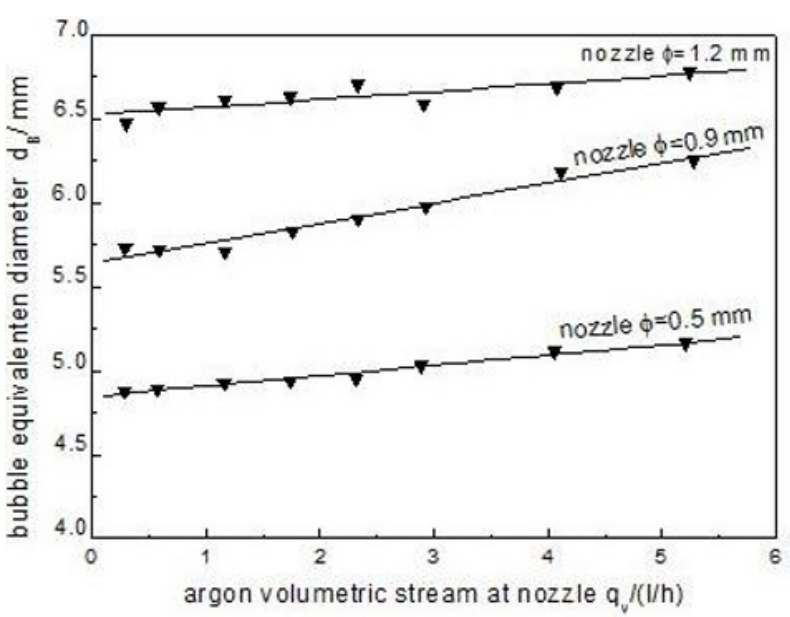

Fig. 8. Dependency of bubble equivalent diameter on volumetric gas stream at nozzle. 


\subsection{The gas phase rising zone}

Most of works assigned to estimate a zone inside which a gas phase is present are based on analyzes of flow patterns obtained from model studies of bubble movement or gas stream, most often observe in a water filled container. It was proved by many investigators [12-16] who were studied the phenomenon's. From the practice point of view in estimation of gas phase flow zone the most important are computer tomography methods utilizing mainly capacitance or optical sensors [17-18].

Ultrasonic echo pulse method enables as well estimation of a zone inside which exists gas flow phase likewise in nontransparent fluids as in liquid metals. A knowledge of the flow region is helpful in fixing the best disposition of argon inlet nozzles in steel refining processes.

Drawings no's $9 \& 10$ present the argon gas rise bubble distribution in a liquid metal along a route $z=$ $157 \mathrm{~mm}$ above inlet nozzle for $B=0 \mathrm{mT} \& B=300 \mathrm{mT}$. On the right side is presented the signal quantity of rise bubbles on each measuring level as well as flow zone width $\Delta X$ on some levels. The measurements has shown, that in magnetic field the argon bubbles rises straightforward and the rise zone is even 50\% smaller than the rise zone without magnetic field. It is clearly visible on a Fig.10. where the flow of argon bubbles divides into two streams at $B=0 \mathrm{mT}$ and at $B=300$ mT. The flow goes up nearly straightforward with a maximum flow zone width $\Delta X=14 \mathrm{~mm}$. The straight forward movement of bubbles bring forth a greater number of bubbles into the ultrasonic region of measuring sensor as it is salient for regions higher than $82 \mathrm{~mm}$ above nozzle. There the number of bubbles found in the ultrasonic field of the sensor is a couple of times greater than in a flow without a magnetic field.

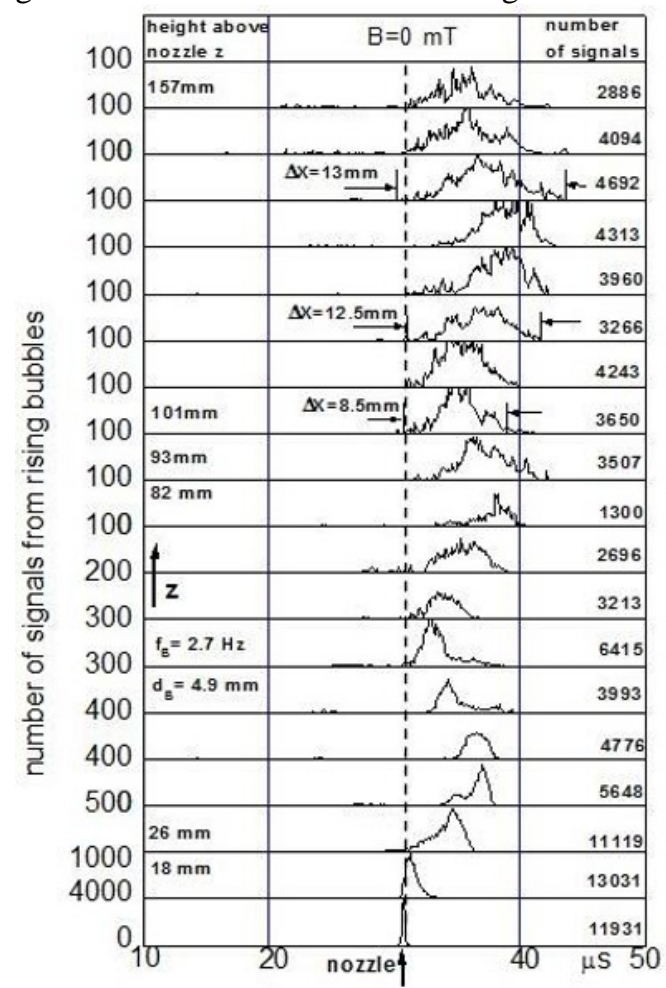

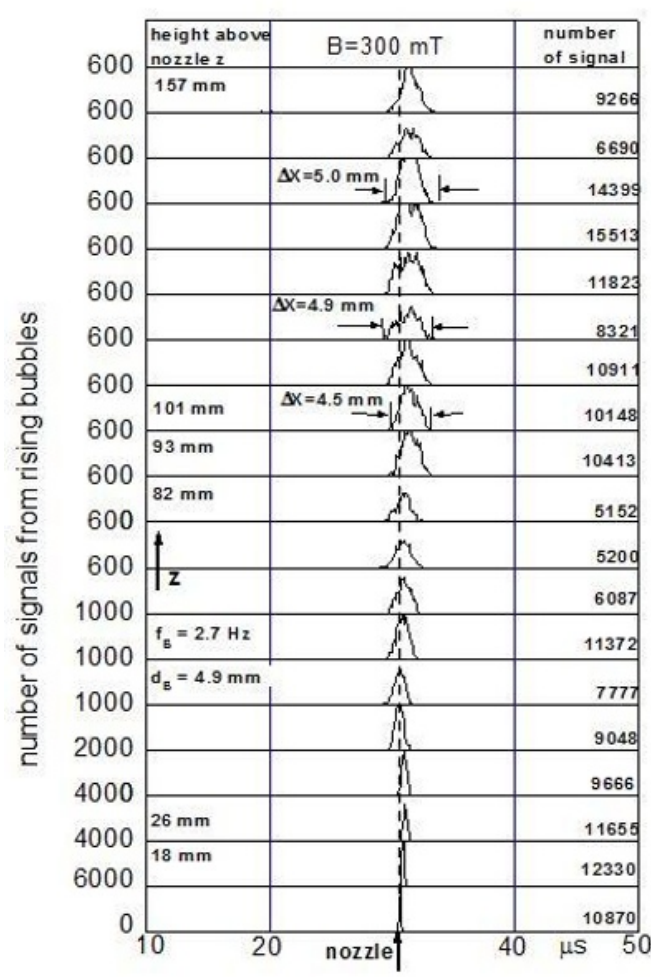

Fig. 9. Rising gas bubble distribution for nozzle $\phi=0.5 \mathrm{~mm}$ and $q_{\mathrm{v}}=0.6 \mathrm{l} / \mathrm{h}$.

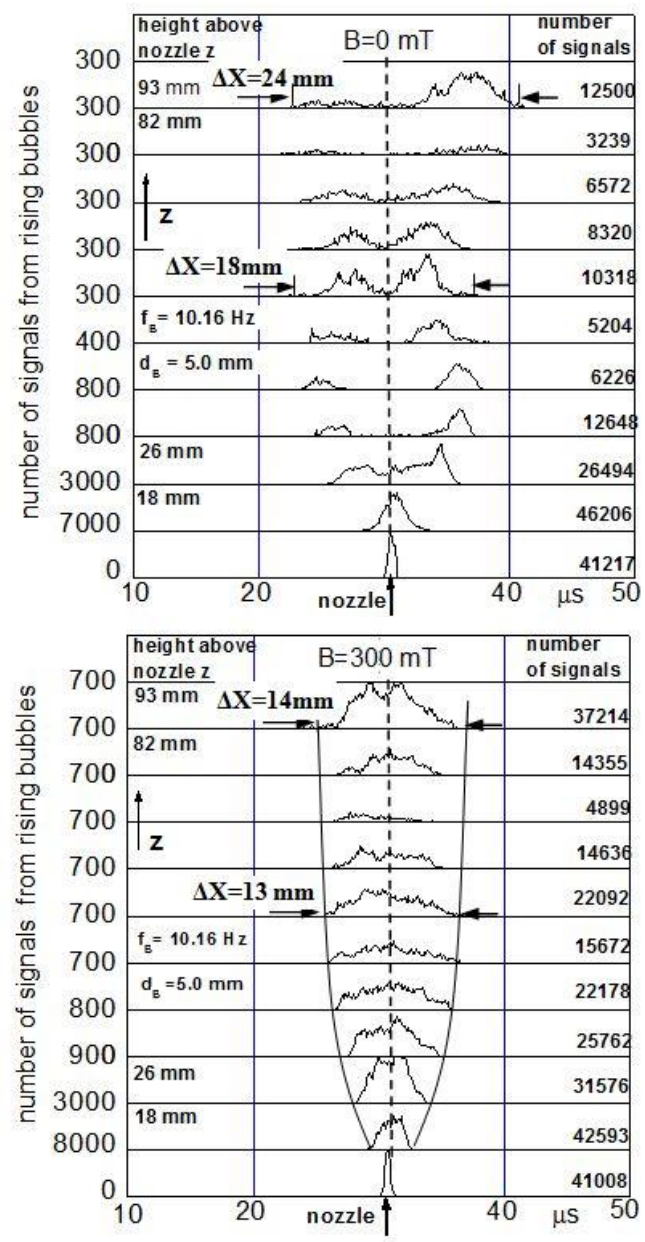

Fig. 10. Rising gas bubble distribution for nozzle $\phi=0.5 \mathrm{~mm}$ and $q_{\mathrm{v}}=2.4 \mathrm{l} / \mathrm{h}$. 


\section{Summary}

The topic of this paper is adaptation of ultrasonic echo pulse method to indentify two-phase liquid metal-gas. Apart from using it in defectoscopy the echo method maybe well used in measurements of characteristic parameters of liquid metal-gas flows. In these studies only a time measurement of echo route from moving bubbles method has been utilized as that method characterize the most favorably signal-to-noise ratio as well as an easy and precise definition of bubble position and by that estimation of the flow zone.

The accomplished measurements and its result analyzes allows to state the following conclusions:

- the Lorentz' force reduces the argon bubbles rise velocity in liquid metal of GaInSn; the influence of Lorentz' force on rise velocity begins with argon flow stream of $q_{\mathrm{v}}=1.8 \mathrm{l} / \mathrm{h}$

- a magnetic field does not change the creation frequency of gas phase bubbles therefore the average equivalent diameter of rising bubble has not change

- a proper values of gas phase creation frequency for different inlet nozzle diameters of gas introduced to liquid metal enables the right estimation of rise bubble dimension and by that the proper sizing of nozzle diameters for a specific processes of reining liquid steel - a decrease of gas bubble rise velocity in a magnetic field increase the contact time of bubbles with liquid metal and in consequence enable the best utilization of blow-in gas

- the magnetic field influence also the bubbles movement trajectory -practically the bubbles rise up straightforward and its flow zone width is smaller than for the rising without the magnetic field; this effect maybe utilized for suitable disposition and selection of distances between argon inlet nozzles in order to obtain the best distribution of gas phase in processes of refinement and degassing liquid metals without forming dead zones in the refining vat.

\section{References}

1. C. Zahng, Liquid metal driven by gas bubbles in a static magnetic field (Dissertationt, Fakultät Maschinenwesen der Technische Universität Dresden, Dresden, 2009)

2. C. Zhang, S. Eckert, G. Gerbeth, International Journal of Multiphase Flow 31 (2005)

3. Z.H.Wang, S.D. Wang, X.Meng,M.J.Ni, International Journal of Multiphase Flow 94 (2017)

4. S.Boden, S. Eckert, G. Gerbeth, M. Simonnet, M.Anderhuber, P. Gardin, (X-ray visualisation of bubble formation and bubble motion in liquid metals. $6^{\text {th }}$ Int. Conf. on Electromagnetic Proc. of Materials, Dresden, 2009)

5. E. Kaiser, Tm - Technisches Messen, 71 (2004)

6. E. Kaiser, Chemie Ingenieur Technik 76 (2004)

7. H.-D.Sommerlatt, E. Kaiser, Tm - Technisches Messen 71 (2004)
8. A. Andruszkiewicz, K. Eckert, S. Eckert, S. Odenbach, European Physical Journal Special Topics 220 (2013)

9. H. Brauer, Grundlagen der Einphasen -und Mehrphasenströmungen (Verlag Sauerländer, Aarau und Frankfurt am Main, 1971)

10. A. Andruszkiewicz, H.-D. Sommerlatt, Chemical and Process Engineering 29 (2008)

11. N. Kantarci, F. Borek, K.O. Ulgen, Process Biochemistry 40 (2005)

12. A.Sokolichin, C. Eingenberger, A. Lapin, A. Lübbert, Chemical Engineering Science 52 (1997),

13. A.Sokolichin, C.Eingenberger, Chemical Engineering Science 49 (1994)

14. Y. Pan, M.P Dudukovic, AICHE 46, (2000)

15. N.G. Deen, T. Solberg, B.H. Hjertager, Chemical Engineering Science 56 (2001)

16. A. Tomiyama, I. Zun, H. Higaki, Y. Makino, T. Sakaquichi, Nuclear Engineering and Design 175 (1997)

17. $2^{\text {and }}$ International symposium an Process Tomography in Poland $11^{\text {th }}-12^{\text {th }}$ September 2001

18. $4^{\text {and }}$ International symposium an Process Tomography in Poland (Computer Engineering Department Technical University Lodz, September 2004) 